\title{
Ces ondes qui nous menacent. Perceptions profanes des risques associés à quatre dispositifs émettant des ondes électromagnétiques
}

\author{
Patrick Peretti-Watel ${ }^{1}$, Chantal Vergélys ${ }^{2}$, Béatrice Hammer ${ }^{3}$ \\ 1 Sociologue, INSERM-IRD - Université de la Méditerranée, UMR 912 Sciences économiques et sociales, systèmes de santé, \\ sociétés, 13006 Marseille, France \\ 2 Sociologue, Observatoire régional de la santé Provence-Alpes-Côte d'Azur, 13006 Marseille, France \\ 3 Sociologue, EDF-GRETS, 92141 Clamart cedex, France
}

Les hiatus entre les discours scientifiques sur des phénomènes qui concernent directement les populations et les perceptions que celles-ci en ont prennent de plus en plus d'importance dans des sociétés où les applications des connaissances scientifiques interviennent de façon croissante dans la vie quotidienne. Cet article contribue à une analyse des rapports entre sciences et société, thème central pour NSS, en montrant que la compréhension de ces décalages passe par une prise en considération de la cohérence propre des conceptions de chacun.

La Rédaction

\section{Mots-clés :}

santé ; perception des risques; ondes électromagnétiques ; sociologie ; France

\begin{abstract}
Résumé - Cet article étudie les perceptions des risques associés à quatre dispositifs émettant des ondes électromagnétiques, les lignes à très haute tension (THT), les téléviseurs, les téléphones portables et les fours à micro-ondes, à partir du Baromètre Environnement EDF-R\&D 2004. De nombreuses personnes s'inquiètent de l'impact des ondes électromagnétiques sur notre santé : elles interféreraient avec notre électricité naturelle, dérégleraient notre système nerveux, provoqueraient des cancers... Ces opinions s'appuient sur des conceptions profanes du corps, de la santé et de la maladie, et mobilisent diverses sources, plus ou moins légitimes, du discours expert à l'intuition personnelle. L'analyse statistique a mis en évidence un phénomène de cumul des risques perçus : les enquêtés qui pensent qu'un dispositif émetteur d'ondes est dangereux ont tendance à penser de même pour d'autres dispositifs. Enfin, les risques perçus pour ces différents dispositifs partagent des déterminants similaires. Ces résultats suggèrent l'existence d'un cadre perceptif commun, qui présiderait à la façon dont nous percevons tout risque induit par des ondes. Ces perceptions ne sont pas réductibles à de "fausses croyances ": elles mobilisent des conceptions cohérentes du corps, de la santé et de la maladie, elles se nourrissent du nivellement contemporain des savoirs, et elles révèlent un rapport conflictuel des individus aux transformations que connaissent les sociétés contemporaines.
\end{abstract}

\section{Keywords:}

Abstract - Electromagnetic threats. Lay risk perceptions related to four technological devices health; risk perception; electromagnetic fields; sociology; France diffusing electromagnetic fields. Thanks to data from the French Environmental Barometer EDF-R\&D 2004 (national representative sample of French aged over 15), this paper investigated lay risk perceptions related to exposure to electromagnetic fields (from an electric power-line, a TV, a cellular phone or micro-wave oven). Answers to an opened-ended question suggested that many people worry about such exposure: it may interfere with natural electricity circulating in the human body, disturb the nervous system and cause cancer. These answers revealed a conception of human body, health and disease, and participants referred to various kinds of arguments, from expert knowledge to personal intuition. Statistical analyses showed that risk perceptions related to these four devices were strongly correlated with each other and shared common predictors. These results suggest that these risk perceptions are

Auteur correspondant : C. Vergélys, chantal.vergelys@laposte.net 
built within the same perceptive frame. One should not consider them as "false beliefs": they are based on consistent conceptions of the body, health and disease, they are fuelled by the contemporary levelling of knowledge, and they reflect the difficulties experienced by many people living in a changing world.

\section{Introduction}

Dans Risk Society, Towards a New Modernity ${ }^{1}$, le sociologue allemand Ulrich Beck caractérise les risques contemporains : il s'agirait selon lui de risques produits par l'homme, qui ne sont plus liés à notre incapacité à contrôler notre environnement naturel, mais au contraire à un « excès » technologique (Beck, 1992). Pour Beck, ces risques technologiques transforment la planète en « siège éjectable », tandis que Giddens, qui le rejoint sur cette analyse, emploie la métaphore du « camion fou » (juggernaut) dans lequel nous serions tous embarqués (Giddens, 1994). Ces risques de grande ampleur et produits par l'homme seraient d'autant plus difficiles à maîtriser qu'ils restent souvent invisibles, silencieux et inodores : nous ne «voyons » pas les radiations radioactives, pas plus que les prions dans notre viande, les gaz à effet de serre dans l'atmosphère, ou les pesticides dans nos aliments.

Cette invisibilité favorise la diffusion de ces risques, dont la mise sur l'agenda social dépend souvent de l'engagement et de la pugnacité de «lanceurs d'alerte » issus de la société civile (Chateauraynaud et Torny, 1999). Elle favorise aussi les controverses scientifiques, ainsi que la volatilité des perceptions profanes: ces risques peuvent être niés, oubliés, ou au contraire dramatisés ; nous nous les « représentons » plus que nous ne les percevons, puisqu'ils échappent à nos sens, sachant que les représentations que nous en forgeons sont souvent éminemment sociales, dans la mesure où elles font intervenir nos préférences, nos valeurs, nos craintes, nos opinions à l'égard de la science, ou encore nos conceptions de la nature ou du corps humain, etc. (Jodelet, 1989 ; Douglas, 1992 ; Peretti-Watel, 2007, 2010).

Le cas des ondes électromagnétiques est emblématique de cette situation. Depuis quelques années, de nombreux objets et installations diffusant des ondes sont suspectés de mettre en danger notre santé : téléviseurs, fours à micro-ondes, lignes à très haute tension, et plus récemment antennes-relais de téléphonie mobile, téléphones mobiles et bornes Wi-Fi. La construction sociale du risque lié aux antennes-relais a d'ailleurs emprunté plusieurs éléments à la mobilisation contre les lignes à très haute tension (Chateauraynaud et Debaz, 2010). Ces risques sont perçus par les simples citoyens comme très incertains, difficiles à observer, ce qui contribue à les rendre menaçants (Slovic, 2000), les rapports d'experts ne parvenant pas à éteindre la polémique (Aurengo, 2009). En

\footnotetext{
1 Ouvrage publié en allemand en 1986 : Risikogesellschaft. Auf dem Weg in eine andere Moderne. Suhrkamp Verlag, Frankfurt am Main.
}

outre, les représentations de ces risques portent la marque des sujets sociaux qui les élaborent. Par exemple, les mobilisations locales suscitées par les antennes-relais ont plus de chances de voir le jour dans des espaces périurbains, qui mélangent des populations hétérogènes et nouvellement installées, qui n'ont donc pas de relation de confiance et de familiarité qui les lieraient avec leur cadre de vie: dans ce cas, la perception du risque porte la marque de la trajectoire résidentielle des individus (Borraz, 2008).

Le présent article s'intéresse aux perceptions profanes des risques associés aux ondes électromagnétiques émises par quatre dispositifs : les lignes à très haute tension (THT), le téléphone portable, le four à micro-ondes et le téléviseur. En mobilisant des données qualitatives (pour les seules lignes THT) et quantitatives, il s'agira de mieux comprendre ces perceptions, et ce qu'elles peuvent avoir en commun ${ }^{2}$.

\section{Les données mobilisées : le Baromètre Environnement}

Les données utilisées sont extraites du Baromètre Environnement EDF-R\&D (2004). Il s'agit d'une enquête postale réalisée tous les deux ans depuis 1990 par 1'Institut TNS Sofres. L'exercice 2004 a été conduit en mars auprès d'un échantillon de 2636 personnes âgées de 15 ans et plus, représentatives de la population française métropolitaine du même âge. Nous avons utilisé les données de 2004, plutôt que celles recueillies en 2006 et 2008, car seule l'enquête de 2004 comportait une question ouverte relative aux effets des lignes THT sur la santé. Le questionnaire de cette enquête aborde des thèmes intéressant directement EDF (économies d'énergie, gestion du parc de centrales nucléaires, développement des économies renouvelables, etc.), en les insérant dans un cadre plus général (perception des risques environnementaux, confiance à l'égard des autorités, opinions sur la science, systèmes de valeurs, etc.). Ne sont prises en considération ici que les réponses aux questions en rapport avec les analyses abordées dans ce texte.

\section{Questions utilisées : l'ouvert et le fermé}

Outre les traditionnelles questions fermées, proposant donc un nombre limité de réponses préformatées, cette

2 Cette recherche a été menée grâce à un financement de la Fondation Santé et Radiofréquences, dans le cadre de l'axe Sciences humaines et sociales de son quatrième appel à projets. 
Tableau 1. Questionnaire des portraits de Schwartz. Questions relatives à la quête du savoir et à la vérité révélée/rationnelle.

Jusqu'à quel point cette personne est-elle comme vous ?*

Items proposés : pas du tout comme moi ; pas comme moi; un petit peu comme moi; un peu comme moi; comme moi; tout à fait comme moi.

Volonté de savoir :

Il/elle pense qu'il faut toujours progresser et approfondir ses connaissances. Ce qui est vrai est important pour lui/elle.

C'est important pour lui/elle de connaître le pourquoi des choses. Il/elle aime réfléchir de manière logique et faire appel à sa raison.

C'est important pour lui/elle de développer son esprit critique. Il/elle aime prendre en compte la complexité des choses.

Vérité révélée/rationnelle :

Il/elle pense que la plupart des choses qui nous arrivent dans la vie ont une signification cachée. Pour cette personne, rien n'arrive par hasard. Cette personne pense que la raison ne mène pas à la vraie connaissance. C'est important pour lui/elle de ne se fier qu'à son instinct pour prendre des décisions importantes.

Il/elle pense qu'il faut chercher le réel authentique au-delà des apparences et du quantifiable. Des pratiques comme la magie, la voyance ou l'astrologie sont importantes pour lui/elle.

*La formulation adoptée, dite " des portraits », souvent utilisée en psychométrie, est censée faciliter les réponses en distanciant l'enquêté de l'énoncé : on ne lui demande pas s'il possède tel ou tel trait, mais s'il ressemble à une personne le possédant.

enquête comprenait également une question ouverte. En effet, les personnes qui répondaient « oui » à la question «Selon vous, la présence de lignes électriques à haute tension peut-elle avoir des conséquences sur l'état de santé des gens qui habitent à proximité ? » étaient ensuite invitées à rédiger elles-mêmes un commentaire de quelques mots ou de quelques lignes, pour préciser leur point de vue sur les dangers des lignes THT.

Trois autres questions fermées portaient sur la perception du risque associé à trois formes d'exposition à des ondes électromagnétiques. Elles avaient une formulation identique : « Pouvez-vous me dire si vous pensez qu'il est ou non dangereux pour la santé... d'utiliser un téléphone portable ; de rester plusieurs heures par jour à côté d'une télévision allumée ; de manger des aliments réchauffés au four à micro-ondes ? » Elles comportaient trois modalités de réponse : « oui », « non » et « je ne sais pas ».

Pour étudier les déterminants des réponses à ces questions, outre les caractéristiques sociodémographiques traditionnelles (sexe, âge, diplôme, catégorie socioprofessionnelle de la personne de référence du foyer), le questionnaire permettait aussi de s'intéresser aux attitudes des enquêtés à l'égard du savoir et de la connaissance. Pour exploiter ces réponses, nous avons utilisé deux échelles validées de psychologie sociale relatives à l'étude des systèmes de valeurs (Schwartz et Bilsky, 1990 ; Wach et Hammer, 2003). La première mesure la «volonté de savoir ", tandis que la seconde distingue les individus selon qu'ils adhèrent à une conception du savoir et de la vérité plus ou moins en accord avec la conception cartésienne dominante ("vérité rationnelle» versus "vérité révélée »). Le détail des questions posées et des items de réponse se trouve dans le tableau 1.
Pour chacune de ces deux dimensions, les réponses aux trois questions correspondantes ont été recodées numériquement de 1 («pas du tout comme moi ») à 6 («tout à fait comme moi ») puis sommées, pour obtenir ainsi deux scores variant de 3 à 18. Plus le premier score est élevé, plus l'enquêté exprime une volonté de comprendre le monde qui l'entoure, et plus le second score est élevé, plus l'enquêté adhère à une conception de la vérité " révélée » plutôt que " rationnelle ».

Enfin, pour compléter ces deux scores, nous avons également utilisé les réponses (« oui », « non » et « je ne sais pas ») à une question destinée à repérer parmi les enquêtés ceux qui valident des formes de connaissance qui se situent clairement en marge de la science légitime et institutionnelle, sachant que cette question a une parenté avec les questions relatives à d'électromagnétisme : « D’après vous, certaines personnes ont-elles vraiment le pouvoir de contrôler l'énergie électromagnétique (magnétiseurs, etc.) ? »

\section{Stratégie d'analyse : recodages et exploitation statistique}

Nous avons d'abord réalisé une analyse thématique des réponses à la question ouverte, dans laquelle les personnes enquêtées commentaient leur réponse positive quant aux effets des lignes THT sur la santé. Nous attendions des regroupements des réponses par thèmes qu'ils nous aident à nourrir l'interprétation des analyses statistiques réalisées par la suite. Puis, nous avons étudié les relations statistiques entre les perceptions des risques liés aux quatre dispositifs exposant aux ondes : ligne THT, téléphone portable, télévision, four à micro-ondes. L'analyse statistique parallèle des déterminants de ces 
quatre perceptions s'est faite en deux temps, avec des analyses bivariées, puis des régressions logistiques dichotomiques complémentaires ${ }^{3}$ (en opposant les « oui » aux « non » et aux « ne sait pas » regroupés), avec sélection pas à pas ascendante des covariables les plus significatives (seuil de sélection $p<0,05$ ) [Tab. 2 et 3].

\section{Résultats : quels risques perçus pour les lignes THT ?}

\section{Analyse thématique des réponses à la question ouverte}

À la question: "Selon vous, la présence de lignes électriques à haute tension peut-elle avoir des conséquences sur l'état de santé des gens qui habitent à proximité ? ", $39 \%$ des personnes interrogées (soit 1028 personnes) ont répondu « oui », $15 \%$ ont répondu «non », tandis que $46 \%$ ont choisi l'item « je ne sais pas ». Parmi les 1028 enquêtés qui étaient invités à répondre à la question ouverte qui suivait, pour expliquer leur réponse positive, 845 (soit $82 \%$ ) ont rédigé une réponse. Ces réponses peuvent être examinées sous trois aspects, fréquemment combinés dans une même réponse : d'abord, le médium par lequel agissent les lignes THT (le magnétisme, les ondes, le bruit, etc.) et la façon dont il interfère avec le corps humain, ensuite les conséquences proprement dites (maladies, fatigue, etc.), enfin les sources sur lesquelles s'appuie éventuellement l'enquêté, et son degré de certitude.

\section{Des ondes qui interféreraient avec notre électricité naturelle}

Trois des biais par lesquels les lignes électriques à haute tension peuvent avoir des effets sur la santé s'avèrent très minoritaires dans les réponses : les accidents (envisagés par 26 enquêtés, qui mentionnent par exemple : « le risque d'électrocution consécutif à la rupture d'une ligne»), les nuisances esthétiques (citées par

\footnotetext{
3 L'analyse bivariée consiste à étudier la relation statistique entre deux variables, par exemple le vote et l'âge. L'analyse multivariée consiste à considérer simultanément les relations entre une variable " expliquée » et plusieurs variables " explicatives ", afin de tenir compte des relations entre variables explicatives. Par exemple, la relation observée entre le vote et l'âge peut être due au fait que le sexe et le diplôme influencent le vote, et qu'ils sont aussi corrélés à l'âge (les femmes vivent plus longtemps, les nouvelles générations sont plus diplômées). Une analyse multivariée permet alors de mesurer la relation entre vote et âge, en contrôlant les effets du sexe et du diplôme. Lorsque la variable expliquée est dichotomique, la régression logistique dichotomique est la méthode d'analyse multivariée la plus usuelle.
}

13 personnes, par exemple : « le désagrément de la vue de ces lignes qui dégradent le paysage doit saper le moral ») et le bruit (mentionné dans 64 réponses, dont « le grésillement permanent qui finit par devenir très énervant et stressant »).

Les principaux éléments incriminés - et de loin, puisqu'ils sont présents dans 248 commentaires (soit $30 \%$ ) - sont les «ondes», les «rayonnements», les « radiations », les «champs », ou encore les « arcs électriques » ou « magnétiques ». On notera que, comme les nuisances esthétiques et sonores, c'est un médium continu, diffus, mais celui-ci échappe à nos sens. Ces ondes sont qualifiées de "malsaines", «néfastes", «nuisibles", «mauvaises pour les organes internes". Elles «perturbent le fonctionnement de l'organisme», créent des « interférences sur le corps humain », « dérèglent » l'organisme (le « cœur », le « sommeil », le « système immunitaire » et, plus souvent encore, le «système nerveux»), en provoquant des troubles qui sont exprimés par des expressions variées : « désordre électromagnétique», "perturbation de l'horloge biologique», «perturbation du physique et du mental », « dérèglement de la glande thyroïde », " perturbations cardiaques et tensions diverses ", etc. Plusieurs aspects sont évoqués : le corps est conducteur, « sensible aux ondes électromagnétiques : lorsqu'un conducteur est parcouru par un courant électrique, celui-ci crée un champ électrique et un champ magnétique qui peuvent engendrer des effets nocifs sur l'organisme ; il en est de même des lignes électriques à haute tension en plus fort ». Le courant qui circule dans les lignes THT «excite les nerfs : les gens sont survoltés et ils pètent les plombs », "ces lignes envoient dans l'atmosphère une très grande quantité d'électricité, les gens ressentent cette électricité et sont souvent irritables, énervés allant jusqu'à la dépression ». Cette sensibilité est d'autant plus forte que le corps luimême est électrique, puisque l'influx nerveux s'y propage tel un signal électrique, d'où de " sérieuses interférences entre l'électricité humaine et ce monde THT » et une «perturbation du fonctionnement électrique du corps (transmission de l'influx nerveux) due aux radiations électromagnétiques de la ligne ». Il est dit encore que « les champs magnétiques interfèrent sur le fonctionnement général des activités électriques du corps, donc possibilité de symptômes variés » et qu'«il est reconnu que les émanations d'ondes et les interférences induites par ces lignes perturbent le corps humain, étant nousmêmes sous influx électriques ».

Toutefois, ces ondes et autres radiations ne se contentent pas de perturber le fonctionnement interne des individus. Non contentes de modifier la mécanique corporelle, elles en altéreraient aussi les composantes élémentaires : «modification de la multiplication cellulaire », «cellules agressées par les ondes électromagnétiques sans arrêt », « désordre physico-chimique », «les 
Tableau 2. Facteurs associés au fait de juger dangereux pour la santé (« oui » versus «non » et « ne sait pas ») quatre dispositifs émetteurs d'ondes, analyses bivariées.

\begin{tabular}{|c|c|c|c|c|}
\hline & $\begin{array}{l}\text { Habiter près d'une } \\
\text { ligne THT }\end{array}$ & $\begin{array}{l}\text { Rester près d'un } \\
\text { téléviseur allumé }\end{array}$ & $\begin{array}{l}\text { Utiliser un } \\
\text { téléphone } \\
\text { portable }\end{array}$ & $\begin{array}{c}\text { Manger } \\
\text { des aliments } \\
\text { réchauffés au four à } \\
\text { micro-ondes }\end{array}$ \\
\hline & \multicolumn{4}{|c|}{ \% en ligne } \\
\hline Sexe: & & & & \\
\hline - homme & 37 & 34 & 31 & 10 \\
\hline - femme & $39 \mathrm{~ns}$ & $35 \mathrm{~ns}$ & $34 \mathrm{~ns}$ & $10 \mathrm{~ns}$ \\
\hline \multicolumn{5}{|l|}{ Âge : } \\
\hline$-15-24$ & 32 & 28 & 36 & 14 \\
\hline$-25-34$ & 47 & 25 & 33 & 9 \\
\hline$-35-49$ & 44 & 32 & 34 & 9 \\
\hline$-50-64$ & 39 & 37 & 31 & 10 \\
\hline-65 ans et plus & $28^{* * *}$ & $46^{* * *}$ & $31 *$ & 10 * \\
\hline \multicolumn{5}{|l|}{ Diplôme : } \\
\hline - études primaires, BEPC & 27 & 35 & 36 & 13 \\
\hline - CAP, BEP, bac pro & 38 & 34 & 31 & 11 \\
\hline - IUT, BTS, $1^{\mathrm{er}}$ cycle supérieur & 44 & 33 & 31 & 7 \\
\hline$-2^{\mathrm{e}}$ et $3^{\mathrm{e}}$ cycles & $48^{* * *}$ & $36 \mathrm{~ns}$ & $36 \mathrm{~ns}$ & $9 * *$ \\
\hline \multicolumn{5}{|l|}{$\begin{array}{l}\text { Catégorie socioprofessionnelle du chef de } \\
\text { ménage : }\end{array}$} \\
\hline - agriculteur exploitant & 25 & 26 & 21 & 12 \\
\hline - artisan, commerçant, chef d'entreprise & 53 & 35 & 38 & 11 \\
\hline - cadre, profession intellectuelle sup. & 46 & 28 & 31 & 7 \\
\hline - profession intermédiaire & 46 & 32 & 31 & 7 \\
\hline - employé & 40 & 32 & 33 & 9 \\
\hline - ouvrier (réf.) & 37 & 32 & 34 & 14 \\
\hline - retraité & 30 & 42 & 31 & 9 \\
\hline - autre inactif & $36^{* * *}$ & $32^{* * *}$ & $34 \mathrm{~ns}$ & 12 * \\
\hline \multicolumn{5}{|l|}{$\begin{array}{l}\text { Certaines personnes ont le pouvoir de } \\
\text { contrôler l'énergie électromagnétique : }\end{array}$} \\
\hline- oui & 34 & 32 & 31 & 9 \\
\hline - non, ne sait pas & $49^{* * *}$ & $38^{* *}$ & $37^{* *}$ & $13^{* *}$ \\
\hline Score à l'échelle « volonté de savoir »\# & 13,1 vs $12,3^{* * *}$ & 12,9 vs $12,5^{* * *}$ & 12,8 vs $12,6^{*}$ & 12,7 vs $12,7 \mathrm{~ns}$ \\
\hline Score à l'échelle « vérité révélée »\# & 8,6 vs $8,3^{* *}$ & 8,5 vs 8,4 ns & 8,7 vs $8,3^{* * *}$ & 9,3 vs $8,3^{* * *}$ \\
\hline
\end{tabular}

${ }_{* * *}^{* * *},{ }^{*}, \mathrm{~ns}$ : respectivement significatif à $p<0,001, p<0,01, p<0,05$, non significatif. Test utilisé: $\chi^{2}$ de Pearson.

\# : pour les deux scores, qui sont des variables quantitatives, sont indiqués les scores moyens associés aux modalités " oui » d'une part, «non » et « ne sait pas » d'autre part, avec un test de Student d'égalité de ces moyennes.

Source : Baromètre Environnement EDF-R\&D 2004.

lignes électriques de haute tension créent des champs magnétiques assez importants, or les champs magnétiques peuvent polariser des molécules de nos cellules».

\section{Quels effets sur la santé ? Faiblesse, cancer et atteintes du système nerveux}

Parmi les personnes qui ont répondu à la question ouverte, 36 évoquent un « affaiblissement général » de la santé, une «faiblesse chronique », ou encore une " fragilité immunitaire », 37 citent des problèmes cardiaques, tandis que les maladies respiratoires et de la peau sont moins souvent citées. Un effectif similaire (32 enquêtés) envisage quant à lui des problèmes de développement pour les fotus et les enfants en bas âge : « malformations fœtales », «malformations à la naissance», «fausses couches ", « avortements », " on dit que cela empêche de dormir et que c'est mauvais pour les bébés ». Ensuite, 106 personnes mentionnent des problèmes de sommeil, en faisant parfois référence au bruit qui émane des lignes THT, mais en associant le plus souvent cet effet avec des «troubles nerveux ».

En suivant un ordre croissant des citations, viennent ensuite, nettement plus fréquentes (puisque concernant 238 réponses), les inquiétudes dues au caractère supposé «cancérigène » des lignes THT. Sont évoqués divers 
Tableau 3. Facteurs associés au fait de juger dangereux pour la santé (« oui » versus «non » et « ne sait pas ») quatre dispositifs émetteurs d'ondes, régressions logistiques.

\begin{tabular}{|c|c|c|c|c|}
\hline & $\begin{array}{c}\text { Habiter près } \\
\text { d'une ligne } \\
\text { THT }\end{array}$ & $\begin{array}{l}\text { Rester près d'un } \\
\text { téléviseur } \\
\text { allumé }\end{array}$ & $\begin{array}{l}\text { Utiliser un } \\
\text { téléphone } \\
\text { portable }\end{array}$ & $\begin{array}{c}\text { Manger des aliments } \\
\text { réchauffés au four à } \\
\text { micro-ondes }\end{array}$ \\
\hline & \multicolumn{4}{|c|}{ Odds ratios } \\
\hline $\begin{array}{l}\text { Sexe : } \\
\text { - homme } \\
\text { - femme (réf.) }\end{array}$ & NS & NS & NS & NS \\
\hline $\begin{array}{l}\text { Âge : } \\
-15-24 \\
-25-34 \\
-35-49 \\
-50-64 \\
-65 \text { ans et plus (réf.) }\end{array}$ & $\begin{array}{l}1,0 \mathrm{~ns} \\
1,7^{* * *} \\
1,7^{* * *} \\
1,4^{* *} \\
-1-\end{array}$ & $\begin{array}{l}0,4^{* * * *} \\
0,4^{* * *} \\
0,5^{* * *} \\
0,7^{* *} \\
-1-\end{array}$ & NS & NS \\
\hline $\begin{array}{l}\text { Diplôme : } \\
\text { - études primaires, BEPC (réf.) } \\
\text { - CAP, BEP, bac pro } \\
\text { - IUT, BTS, } 1^{\mathrm{er}} \text { cycle supérieur } \\
-2^{\mathrm{e}} \text { et } 3^{\mathrm{e}} \text { cycles }\end{array}$ & $\begin{array}{l}-1- \\
1,3 * \\
1,6 \text { ** } \\
1,7 \text { ** }\end{array}$ & NS & NS & $\begin{array}{l}-1- \\
0,8 \mathrm{~ns} \\
0,5^{* *} \\
0,8 \mathrm{~ns}\end{array}$ \\
\hline $\begin{array}{l}\text { Catégorie socioprofessionnelle du chef de ménage : } \\
\text { - agriculteur exploitant } \\
\text { - artisan, commerçant, chef d'entreprise } \\
\text { - cadre, profession intellectuelle supérieure } \\
\text { - profession intermédiaire } \\
\text { - employé } \\
\text { - ouvrier (réf.) } \\
\text { - retraité } \\
\text { - autre inactif }\end{array}$ & NS & NS & $\begin{array}{c}0,7 \mathrm{~ns} \\
1,3 \mathrm{~ns} \\
0,8 \mathrm{~ns} \\
0,7^{*} \\
0,8 \mathrm{~ns} \\
-1- \\
0,9 \mathrm{~ns} \\
1,0 \mathrm{~ns}\end{array}$ & NS \\
\hline $\begin{array}{l}\text { Certaines personnes ont le pouvoir de contrôler } \\
\text { l'énergie électromagnétique : }\end{array}$ & & & & \\
\hline $\begin{array}{l}\text { - oui } \\
\text { - non, ne sait pas }\end{array}$ & $\begin{array}{l}1,7^{* * *} \\
-1-\end{array}$ & $\begin{array}{l}1,4^{* * *} \\
-1-\end{array}$ & $\begin{array}{l}1,3 * \\
-1-\end{array}$ & $\begin{array}{l}1,3 * \\
-1-\end{array}$ \\
\hline Score à l'échelle « volonté de savoir » & $1,1^{* * *}$ & $1,1^{* * *}$ & NS & NS \\
\hline Score à l'échelle « vérité révélée » & $1,1^{*}$ & NS & $1,1^{* *}$ & $1,1^{* * *}$ \\
\hline
\end{tabular}

***,,$* * *$, ns : respectivement significatif à $p<0,001, p<0,01, p<0,05$, non significatif. Test utilisé: $\chi^{2}$ de Wald. réf. : modalité de référence pour la régression logistique (odds ratio fixé à 1 ).

NS : variable non sélectionnée par la procédure pas à pas ascendante.

Source : Baromètre Environnement EDF-R\&D 2004.

cancers (de la peau, des os, de la thyroïde, leucémie), mais au premier rang figurent les tumeurs du cerveau, souvent associées aux altérations cellulaires que provoqueraient les ondes : « les ondes électriques peuvent être à l'origine de tumeurs et de perturbations du système nerveux », « le magnétisme électrique peut provoquer des altérations cérébrales voire une tumeur cérébrale », « rayonnement du champ magnétique altérant les cellules du cerveau et favorisant le développement des tumeurs ».

Les effets sanitaires néfastes les plus cités (387 personnes) sont les atteintes au système nerveux (en dehors des tumeurs cérébrales déjà citées). La majeure partie des citations en la matière peuvent être classées dans quatre rubriques : problèmes de nervosité, d'anxiété, de stress ou d'irritabilité (159 enquêtés), maux de tête, céphalées, migraines (115), problèmes au cerveau, y compris la maladie d'Alzheimer (32), et enfin dépression (17).

\section{Des avis plus ou moins autorisés}

Une trentaine des personnes enquêtées appuient leur dire sur leur propre expérience ou sur celle de proches ( "maux de tête pour y avoir déjà goûté », "je connais personnellement des personnes ayant travaillé des années sous une ligne THT : à la retraite l'épouse a fait 


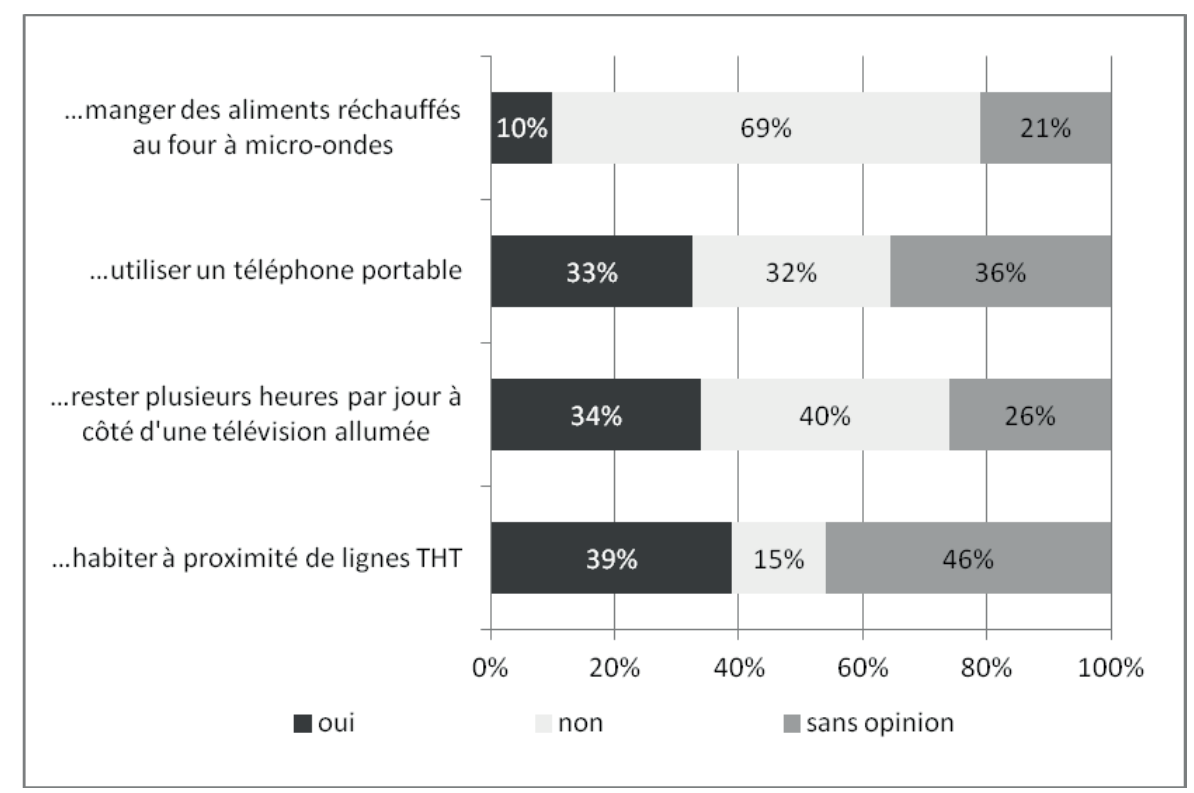

Fig. 1. Perception du risque associé à divers comportements ou situations : est-il dangereux pour la santé de... (d'après le Baromètre Environnement EDF-R\&D 2004).

une grave dépression nerveuse et le mari à des problèmes psychiatriques»), ou se fient à leur intuition tout en avouant ne pas être en mesure de s'expliquer (« il s'agit d'une conviction, mais que je ne peux expliquer », « je ne sais pas vraiment mais je suis sûre qu'il y a des conséquences ", « je ne sais pas, mais les champs magnétiques ne peuvent pas être bénins à mon avis»). En revanche, une cinquantaine de réponses se réclament d'une source d'autorité pour appuyer leur opinion, sachant que cette source est le plus souvent anonyme : «il a toujours été dit », « il est bien connu », «c'est prouvé », etc. Quelques enquêtés citent aussi les médias : télévision, radio, presse écrite, etc. D'autres mentionnent des sources expertes, par exemple "plusieurs études sérieuses ont montré le lien qui existe entre certains cancers et la proximité de lignes THT ». Toutefois les enquêtés qui se réfèrent aux experts soulignent souvent les incertitudes dans ce domaine : «on n'a justement pas le recul nécessaire pour connaître tous les risques, mais il apparaît que le taux de certaines maladies est plus grand à proximité », « il y a différents cancers soit déclenchés, soit accélérés par ces lignes, mais là les scientifiques ne sont pas d'accord ».

Pour étayer leur opinion, certaines personnes mobilisent plus particulièrement deux formes d'analogie. L'une passe par une référence aux plantes et aux animaux : les effets néfastes des lignes THT sur eux étant prouvés, il y a tout lieu de penser que ce qui vaut pour eux vaut aussi pour les hommes : « le champ électrique est néfaste aux plantes : donc à l'être humain ». Certains invoquent à ce propos leur propre expérience : «j'ai pu constater le nondéveloppement d'arbres situés sous une ligne haute tension ; si le risque existe sur les végétaux, pourquoi n'existerait-il pas sur l'homme », « dans les terres cultivées, on constate des différences sous une ligne, les ondes dégagées influent sur la nature, donc sur l'homme». La seconde analogie passe par une similitude établie avec les téléphones mobiles et les antennes-relais : "émettre des ondes, ce n'est pas bon pour la santé, il suffit de voir les téléphones portables! ", créant des " champs électromagnétiques (comme les relais radiotéléphone) », les lignes THT « ont les mêmes influences néfastes que les ondes téléphoniques, elles occasionnent des maux de tête et donnent un sentiment de malaise!», elles ont «les mêmes [effets] que pour les champs électromagnétiques des antennes: effets cancérigènes, démangeaisons, eczéma, avortement, baisse de l'immunité, baisse des anticorps et du fer, céphalées, insomnies, acouphènes, phosphènes $»$.

\section{Effets perçus sur la santé de quatre dispositifs émet- teurs d'ondes : le poids des indécis}

La figure 1 détaille les résultats obtenus pour les quatre dispositifs émetteurs d'ondes retenus dans notre analyse. Une première observation à faire porte sur le poids des indécis : il n'est jamais négligeable, puisqu'il va du cinquième à près de la moitié des personnes enquêtées, en passant par le quart et le tiers d'entre elles ; il peut même constituer la majorité relative des réponses (comme dans le cas des lignes THT).

Ensuite, lorsque des opinions sont émises, on note qu'elles sont corrélées positivement entre elles. C'est ce qu'illustre la figure 2. Celle-ci a été construite de la façon suivante : pour chaque dispositif, la proportion de personnes le jugeant dangereux pour la santé a été calculée 


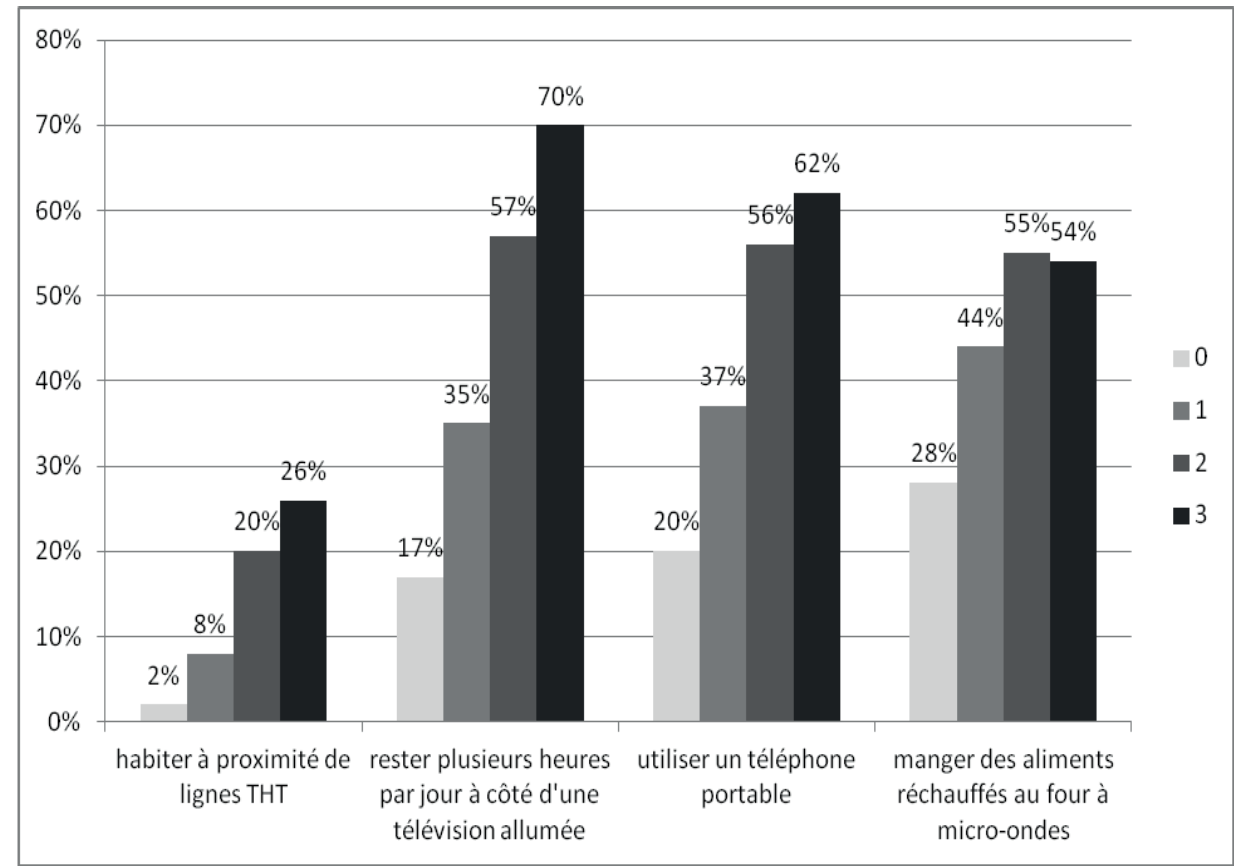

Fig. 2. Cumul des risques perçus concernant quatre dispositifs émettant des ondes (d'après Baromètre Environnement EDF-R\&D 2004).

en fonction du nombre de dispositifs également incriminés parmi les trois autres étudiés ici. Par exemple, parmi les personnes qui ne pensent pas qu'utiliser un four à micro-ondes, habiter près d'une ligne THT ou rester à côté d'une télévision allumée serait dangereux pour la santé (valeur 0 sur la figure 2), $17 \%$ considèrent qu'en revanche il est dangereux d'utiliser un téléphone portable. Parmi les personnes qui jugent dangereux un seul des trois autres dispositifs (ligne THT, four à micro-ondes ou télévision, valeur 1), cette proportion passant à $35 \%$, puis à $57 \%$ pour ceux qui en mettent en cause deux. Enfin, parmi les enquêtés qui estiment que les trois autres dispositifs sont dangereux, $70 \%$ pensent qu'utiliser un téléphone portable est dangereux pour la santé.

Ainsi, il apparaît que, quel que soit le dispositif émetteur d'ondes considéré, les personnes qui considèrent comme dangereux un dispositif sont aussi plus enclines à porter le même jugement sur les autres dispositifs étudiés ici.

\section{Les facteurs associés à la dangerosité perçue : des profils contrastés selon le dispositif considéré}

Le tableau 2 détaille les résultats des analyses statistiques bivariées. Les analyses multivariées sont présentées dans le tableau 3 et ne sont citées ici que lorsque les résultats diffèrent de ceux de l'analyse bivariée. Hormis en ce qui concerne les différences entre hommes et femmes, qui ne sont jamais significatives, les déterminants sociodémographiques de la dangerosité perçue jouent, et leurs effets varient d'un dispositif à l'autre : ce sont les tranches d'âges intermédiaires (25-49 ans) et les individus les plus diplômés qui craignent davantage les lignes à haute tension; l'âge joue en sens inverse selon que l'on se réfère au risque associé à un usage élevé de la télévision (le risque perçu augmente) ou au téléphone portable (il diminue, mais cet effet disparaît dans l'analyse multivariée) ; enfin un niveau scolaire élevé entraîne une baisse du risque perçu à propos des aliments chauffés au four à micro-ondes. Quant à la catégorie socioprofessionnelle, ses effets sont également variables, lorsqu'ils sont significatifs : par exemple, les professions intermédiaires craignent davantage les lignes THT, mais moins les aliments chauffés au four à micro-ondes, que les ouvriers (mais une fois pris en compte les effets du diplôme, en analyse multivariée, la plupart des effets associés à la catégorie socioprofessionnelle cessent d'être significatifs).

En revanche, quel que soit le dispositif considéré, le risque perçu est plus fréquent parmi les personnes qui croient que certaines personnes ont le pouvoir de contrôler l'énergie électromagnétique. Dans une moindre mesure, on observe la même stabilité des résultats pour les deux échelles de valeurs introduites dans l'analyse : la volonté de savoir est associée aux risques perçus pour les lignes THT, les téléviseurs et les téléphones portables 
(pour ces derniers, pas en multivarié), tandis que la conception de la connaissance qui privilégie une vérité " révélée » plutôt que rationnelle est associée à un risque perçu plus fréquent pour chaque dispositif, hormis le téléviseur (Tab. 2).

\section{Discussion : les risques liés aux ondes, un cadre perceptif commun qui porte la marque des sujets}

\section{Près d'un Français sur deux s'inquiète des lignes THT}

Selon les réponses à la question ouverte, au sein de l'échantillon représentatif de la population française âgée de 15 ans et plus étudié ici, près de quatre Français sur dix s'inquiètent de l'impact des ondes électromagnétiques émises par les lignes THT sur notre santé : ces ondes perturberaient notre électricité naturelle, dérégleraient notre système nerveux, et pourraient même altérer nos cellules, provoquant alors des cancers, en particulier des tumeurs cérébrales. Ces opinions s'appuient sur diverses sources, plus ou moins légitimes, du discours expert à l'intuition personnelle. L'analyse statistique a ensuite mis en évidence un phénomène de cumul des risques perçus : les enquêtés qui pensent qu'un dispositif émettant des ondes est dangereux pour la santé ont tendance à porter le même jugement pour les autres dispositifs envisagés ici. Enfin, les risques perçus pour ces différents dispositifs le sont en fonction de déterminants similaires sur le plan cognitif : la croyance dans la capacité qu'auraient certaines personnes de contrôler l'énergie électromagnétique et, dans une moindre mesure, la volonté de savoir et l'adhésion à une conception « révélée » de la vérité.

\section{Limites de l'étude : des données anciennes issues d'un questionnaire fermé}

Avant de discuter nos résultats, il importe d'en rappeler les limites. D'une part, les enquêtes par questionnaire fermé ne permettent qu'une exploration très sommaire des perceptions profanes; cela reste une limite, même si, dans notre cas, nous avons pu mobiliser les réponses données à une question ouverte pour les lignes THT. En revanche, pour les trois autres dispositifs émetteurs d'ondes envisagés ici, nous n'avons aucune information quant aux types d'effets sur la santé que leur associent les personnes interrogées. D'autre part, les données analysées ici sont relativement anciennes: elles ont été recueillies en 2004, donc avant que la controverse sur les effets sanitaires des téléphones mobiles ou des antennes-relais ne prenne l'ampleur que nous lui connaissons aujourd'hui. Des données plus récentes montrent d'ailleurs que les niveaux de risque perçu ont connu des évolutions très sensibles au cours des années 2000 (IRSN, 2010). Toutefois, l'objectif de cette recherche n'était pas de mesurer des niveaux de risque perçu, mais plutôt de comprendre comment les individus construisent leur représentation d'un nouveau risque, en fonction de leur propre situation sociale, et en mobilisant pour cela d'autres représentations, relatives notamment au corps, à la santé et à la maladie : nous pensons que la façon dont sont construites les représentations d'un nouveau risque évolue beaucoup plus lentement au cours du temps que les niveaux de risque perçu.

\section{Un risque perçu qui dépend des conceptions du corps, de la santé et de la maladie}

Les réponses à la question ouverte sont révélatrices des représentations profanes du corps, de la santé et de la maladie, qui sont mises en jeu dans la représentation du risque découlant de l'exposition aux ondes électromagnétiques : le corps humain est conducteur, il est parcouru en continu par une électricité naturelle qui assure son fonctionnement interne. Les fonctions citées renvoient généralement à des flux (circulation du sang, respiration, influx nerveux), les organes évoqués en sont les supports : le cœur, à la fois « pile » de l'organisme et régulateur des flux respiratoire et sanguin, et le cerveau, considéré comme le siège d'une intense activité électrique. Cette conception du corps humain est un mythe, au sens où l'entend Mary Douglas (au même titre qu'il existe des mythes de la nature ou du savoir, $c f$. Douglas, 1992) : ni vraie, ni fausse, elle repose sur des analogies qui mobilisent des résultats scientifiques vulgarisés, mais surtout elle est cohérente, elle fait sens pour les individus et elle les aide à appréhender le monde qui les entoure.

Dans le droit fil de l'analogie électrique, les ondes émises par les lignes THT sont vues comme " survoltant » les individus exposés qui «péteraient les plombs ». Les ondes sont perçues ici comme une source d'électricité artificielle, émise par des objets technologiques, que notre corps absorbe malgré lui, et qui vient interférer avec notre électricité naturelle. On retrouve ici les grands traits de la dialectique profane santé-maladie mise en évidence par les travaux pionniers de Claudine Herzlich : la santé est conçue comme un équilibre interne naturel, qui est perturbé lorsque l'individu est "intoxiqué » par un agent externe, artificiel (aliments traités avec des produits chimiques, air vicié, bruit, ou dans le cas présent, ondes), auquel il est exposé par la société dans laquelle il vit (Herzlich, 1969).

\section{Un cadre perceptif commun pour tous les risques induits par des ondes?}

Cette interprétation dépasse les seules lignes THT. Elle mobilise des conceptions du corps, de la santé, de la 
maladie, qui pourraient également servir de cadre cognitif de référence pour l'appréhension des risques induits par les autres dispositifs émetteurs d'ondes envisagés dans cet article. Ainsi, la question de l'impact des ondes sur la santé peut être considérée comme révélant un rapport conflictuel des individus aux transformations que connaissent les sociétés contemporaines. Les perturbations de notre organisme par les ondes électromagnétiques font en effet écho aux bouleversements de notre mode de vie induits par les nouvelles technologies dont sont issus les dispositifs qui émettent ces ondes.

Il est d'ailleurs révélateur que les effets perçus sur la santé des lignes THT renvoient avant tout au cancer et aux « maladies nerveuses », qui sont les pathologies cardinales de l'imaginaire contemporain de la maladie, le cancer constituant en outre une métaphore privilégiée pour exprimer le désordre, le chaos, qu'il soit social ou biologique (Pinell, 1992 ; Sontag, 1993 ; Ehrenberg, 1998). Ce rapprochement entre les perceptions de divers risques qui ont en commun d'impliquer des ondes émises par des objets technologiques est justement suggéré par le type d'effets envisagés : par exemple, tout comme les lignes THT, les antennes-relais et les téléphones portables sont soupçonnés de provoquer des atteintes au système nerveux et des tumeurs cérébrales (Martha et al., 2006).

Dans notre étude, trois résultats étayent ce rapprochement. D'abord, pour justifier leur opinion sur les effets délétères des lignes THT, certains des enquêtés se réfèrent justement par analogie aux effets qu'ils imputent aux téléphones mobiles et aux antennes-relais. Ensuite, bien que variables selon qu'elles ont trait aux lignes THT, aux téléviseurs, aux téléphones mobiles ou aux fours à micro-ondes (Fig. 1), les perceptions des risques sont étroitement corrélées les unes aux autres et ont tendance à se cumuler (Fig. 2). Enfin, ces quatre perceptions ont un déterminant significatif commun: la croyance en l'existence de personnes capables de contrôler l'énergie électromagnétique, qui constitue en quelque sorte la réciproque de la croyance selon laquelle les ondes auraient une emprise sur nous.

\section{Risque perçu et légitimité des savoirs}

Bien sûr, l'idée que certaines personnes pourraient contrôler l'énergie électromagnétique peut faire sourire, et le fait qu'elle soit corrélée aux risques perçus étudiés ici pourrait contribuer à «disqualifier» ces perceptions. Toutefois, il serait sans doute erroné d'y voir simplement une forme de superstition primitive. En effet, nos sociétés sont caractérisées par un nivellement sans précédent des savoirs. D'une part, la science «officielle » s'est désenchantée elle-même, elle s'est "balkanisée », et souffre aujourd'hui d'une crise de légitimité, ses résultats apparaissant au mieux comme partiels, provisoires et incertains, au pire comme biaisés par des conflits d'intérêts
(Beck, 1992). D'autre part, les sources de savoir « alternatives ", les para-sciences et les sciences occultes se sont " sécularisées », elles ont gagné en respectabilité en même temps que s'estompaient les frontières entre elles et le savoir « légitime » (Tiryakian, 1972 ; Peretti-Watel, 2002).

Ce brouillage des frontières entre savoirs plus ou moins légitimes est bien sûr accentué par le développement d'Internet, qui accélère et amplifie la diffusion des connaissances tout en occultant fréquemment leur origine. En outre, dans une société qui nous exhorte à prendre notre vie en main en mobilisant les savoirs experts disponibles pour anticiper les risques qui nous menacent (Giddens, 1991), chacun tente d'appréhender ces risques en forgeant ses propres croyances, à partir d'informations hétéroclites, souvent simplifiées et déformées.

$C^{\prime}$ est aussi ce qu'illustrent les réponses à la question ouverte sur les lignes THT puisque, pour justifier la mise en cause de ces lignes, les enquêtés citent de multiples sources, incluant des experts non identifiés, les médias, jusqu'à l'intuition personnelle. Enfin, ces analyses trouvent un écho dans nos résultats statistiques, dans la mesure où la volonté de savoir d'une part et la croyance en une vérité révélée plutôt que rationnelle d'autre part s'avèrent étroitement corrélées avec les risques perçus que nous avons étudiés.

En d'autres termes, même si, pour certains experts, les effets sur la santé des ondes électromagnétiques ne sont que des « risques fantômes » (Brauner, 1997), nos résultats suggèrent que la perception de ces risques n'est pas réductible à de fausses croyances ou à une forme d'ignorance. Bien au contraire, elle se développerait dans un cadre de référence commun, qui articule des conceptions cohérentes du corps humain, de la santé et de la maladie, et qui répond à une volonté de savoir. C'est pourquoi, dans ce domaine comme dans d'autres, il est sans doute illusoire d'espérer clore les controverses en diffusant dans l'espace public des connaissances « indiscutables » : mieux vaut tenter de rapprocher, au moins provisoirement, les points de vue de l'expert et du profane, afin de les faire dialoguer (Barthe et Gilbert, 2005).

\section{Références}

Aurengo, A., 2009. Éditorial. Antennes-relais: les ressorts d'une polémique, Santé Publique, 21, 6, 545-546 (http:// www.cairn.info/revue-sante-publique-2009-6-page545.htm, consulté le 14/10/2013).

Barthe, Y., Gilbert, C., 2005. Impuretés et compromis de l'expertise, une difficile reconnaissance: à propos des risques collectifs et des situations d'incertitude, in Dumoulin, L., La Branche, S., Robert, C., Warin, P., Le Recours aux experts: raisons et usages politiques, Grenoble, Presses universitaires de Grenoble, 43-62. 
Beck, U., 1992. Risk Society: Towards a New Modernity, London, Sage Publication.

Borraz, O., 2008. Les Politiques du risque, Paris, Presses de Sciences Po.

Brauner, C., 1997. Les Champs électromagnétiques, un risque fantôme, Zurich, Compagnie Suisse de Réassurance.

Chateauraynaud, F., Debaz, J., 2010. Le partage de l'hypersensible: le surgissement des électro-hypersensibles dans l'espace public, Sciences Sociales et Santé, 28, 3, 5-33 (http:/ / gspr.ehess.free.fr/documents/FC-JD-hypersensibles.pdf, consulté le 14/10/2013).

Chateaureynaud, F., Torny, D., 1999. Les Sombres Précurseurs : une sociologie pragmatique de l'alerte et du risque, Paris, EHESS.

Douglas, M., 1992. Risk and Blam: Essays in Cultural Theory, London, Routledge.

Ehrenberg, A., 1998. La Fatigue d'être soi : dépression et société, Paris, Odile Jacob.

Giddens, A., 1991. Modernity and Self-Identity, Stanford, Stanford University Press.

Giddens, A., 1994. Les Conséquences de la modernité, Paris, L'Harmattan.

Herzlich, C., 1969. Santé et maladie : analyse d'une représentation sociale, Paris, Mouton.

IRSN., 2010. Baromètre IRSN 2010 : la perception des risques et de la sécurité par les Français, Fontenay-aux-Roses, IRSNDSDRE (http://www.irsn.fr/FR/Documents/RA2010/ IRSN_Barometre_2010.pdf, consulté le 14/10/2013).

Jodelet, D., 1989. Représentations sociales: un domaine en expansion, in Jodelet, D. (dir.), Les Représentations sociales, Paris, Puf, 31-60.

Reçu le 28 novembre 2011. Accepté le 7 novembre 2012.
Martha, C., Coulon, M., Souville, M., Griffet J., 2006. Les risques liés à l'usage du téléphone portable et leur représentation médiatique : l'exemple de trois quotidiens français, Santé Publique, 18, 2, 275-288 (http://dx.doi.org/ 10.3917/spub.062.0275, consulté le 14/10/2013).

Peretti-Watel, P., 2002. Sous les étoiles, rien de nouveau? L'horoscope dans les sociétés contemporaines, Revue Française de Sociologie, 43, 1, 3-33 (http://www.persee.fr/ web/revues/home/prescript/article/rfsoc_00352969_2002_num_43_1_5471, consulté le 14/10/2013).

Peretti-Watel, P., 2007. Sociologie $d u$ risque, $3^{\mathrm{e}}$ éd., Paris, Armand Colin.

Peretti-Watel, P., 2010. La Société du risque, $2^{\mathrm{e}}$ éd., Paris, La Découverte.

Pinell, P., 1992. Naissance d'un fléau : histoire de la lutte contre le cancer en France (1890-1940), Paris, Métailié.

Schwartz, S.H., Bilsky, W., 1990. Toward a theory of the universal content and structure of values: Extensions and cross-cultural replications, Journal of Personality and Social Psychology, 58, 5, 878-891, doi/10.1037/0022-3514.58.5.878.

Slovic, P., 2000. The Perception of Risk, London, Earthscan Publications.

Sontag, S., 1993. La Maladie comme métaphore, Paris, Christian Bourgois.

Tiryakian, E., 1972. Toward the sociology of esoteric culture, American Journal of Sociology, 78, 3, 491-512 (http:// www.jstor.org/stable/2776304, consulté le 14/10/2013).

Wach, M., Hammer, B., 2003. La structure des valeurs est-elle universelle ?: genèse et validation du modèle compréhensif de Schwartz, Paris, L'Harmattan. 